\title{
Thermal Adjustments in Cardiac and Skeletal Muscles of Lizards
}

\author{
Paul Licht \\ Department of Zoology, University of California \\ Berkeley, California \\ WILLIAM R. DAWSON \\ Department of Zoology, University of Michigan \\ Ann Arbor, Michigan \\ VAUGHax H. Shommaker \\ Department of Life Sciences, University of California \\ Riverside, California \\ Received July 7, 1969
}

Summary. I. Isometric twitch tension development was measured over a wide range of temperatures in skeletal and cardiac (ventricular) muscles from diverse Australian lizards representing several families and including both diurnal and nocturnal species.

2. The temperatures at which maximal twitch tension develops (optimal temperatures) in the skeletal muscles is higher, by about $10-15^{\circ} \mathrm{C}$, than in the ventricular muscles from the same species. Resistance to heat damage (thermostability) is similar for the two tissues.

3. Interspecific divergence is evident in the optimal temperatures and thermostabilities of both muscles, and these tissue differences parallel the preferred body temperatures of the species from which they are taken. In several nocturnal lizards (geckos) that have high preferred body temperatures, the thermal adjustments of the muscles resemble those of diurnal species, whereas muscles from geckos with low preferenda have relatively low optimal temperatures.

4. Variations in the heat resistance of the tissues correlate with variations in organismal heat resistance and in general, lethal temperatures for excised muscles and organism are similar.

5. These data are interpreted as indicating that divergence in the thermal adjustments for contractility of muscular tissues are most directly associated with the temperatures characteristically maintained through the behavior of the species rather than to the general geographical (climatic) distribution or phylogenetic position of the species.

\section{Introduction}

The contractile performance of both skeletal and cardiac muscle of several lizards has been investigated over a wide range of temperatures. Marked interspecific differences are apparent in the temperature dependence of contractility, but the basis of these differences is not entirely clear. 
It has been suggested that they are primarily related to variations in thermal preferences and heat resistance of the lizards (Lіснт, 1964, 1967; Dawson, 1967). However, insufficient comparative data are available to rule out the possibility that phylogenetic position independent of thermal considerations may underlie these differences in tissue responses.

Variations in thermal relations are well documented within as well as between families of lizards. Study of properly selected additional species should, therefore, allow assessment of the extent of divergence in tissue responses to temperature within families and of convergence of such responses between families. This information appears fundamental to an understanding of the correlation between the thermal relations of the intact animal and the thermal dependence of its tissue performance.

Existing observations on cardiac and skeletal muscle have been obtained in independent series of observations (for example, DAwson and Bartholomew, 1958 ; Dawson, 1960, 1967 ; LICHT, 1964). The data indicate that the temperatures for maximal tension development in vitro are consistently higher for skeletal muscle than for cardiac muscle from the same species. Since these data were obtained from different individuals and studied under somewhat different conditions, it has up to now not been clear wether or not these observations reflect inherent differences in the tissues.

We undertook a study of a variety of Australian lizards with the above considerations in mind. This allowed us to obtain information on an additional species within one family (Scincidae) for which some information was already available and to extend observations to include several species in two families (Agamidae and Gekkonidae) not previously studied. The array of species with which we dealt differ more conspicuously in their thermal relations than the American species examined previously. The samples of cardiac and skeletal muscle utilized from these animals were taken from the same individuals and studied under closely similar experimental conditions, allowing critical examination of whether or not the thermal dependence of isometric twitch tension really does differ between those tissues in the same organism.

\section{Materials and Methods}

\section{Experimental Animals}

Representatives of the following species were used in this study: Gehyra punctata, Phyllurus milii, and Diplodactylus spinigerus scalaris (Family Gekkonidae); Egernia carinata (Family Scincidae); and Physignathus longirostris, Amphibolurus inermis and A. ornatus (Family Agamidae). Adults of both sexes were used. All were studied during late October to early December (austral spring) except the Diplodactylus, which were studied in April. Observations on the muscles were made within 7-10 days of capture of the lizards. On return to the laboratory they were all held with water but no food at $25^{\circ} \mathrm{C}$. 
All lizards were colleeted in Western Australia; the A. ornatus and E. carinata were obtained in the vicinity of Perth; the Diplodactylus near Port Hedland (Mundabullangana Station); the Gehyra near Meekatharra; the A. inermis near Hamelin Pool; and the Physignathus on the Murchison River near the coastal highway. The total patterns of distribution in Australia and details of the habitats in which these species occur is provided by Licht et al. (1966a). The three geckos are entirely crepuscular or nocturnal in habit and the skinks and agamids are all diurnal.

\section{Muscle Contractility}

The preparation of skeletal muscle (m. pubioischiotibialis) and the procedures for measuring their contractility at different temperatures were similar to those used by LICнт (1964). Muscles were excised and mounted vertically in a well oxygenated Ringer's solution between a pair of platinum ring electrodes; the electrodes did not contact the muscle directly. Contractility was measured as isometric tension development during single, widely spaced twitches produced by rectangular wave pulses delivered by a Grass Model S-4C stimulator. Tension was recorded using a mechanoelectrical transducer (RCA 5734) in conjunction with a Sanborn Recording Oscillograph (Model 299).

Individual museles were tested at intervals of approximately $4^{\circ} \mathrm{C}$, proceeding from cool to warm conditions. The tissues were allowed to equilibrate for five minutes at each temperature before stimulation. Stimuli (voltage and duration) were then adjusted to produce maximal twitch tension development at each temperature. Museles were returned to a reference temperature $\left(24^{\circ} \mathrm{C}\right)$ for five minutes between each test temperature and then stimulated to ascertain the extent of deterioration in performance (i.e., fatigue or irreversible heat damage). The tension developed at each test temperature was expressed as a percentage of the preceding reference tension. The maximum percentage was then taken as $100 \%$ and the others expressed as a fraction of this value.

Excised ventricles were used to determine performance in cardiac muscle. These were prepared in the manner described by DAwSON and BARTHOLOMEW (1958) and the same general protocol followed; this was similar to that described above for skeletal muscle. In the present study, a higher concentration of sodium chloride was used than in the earlier studies with ventricular tissue ( $155 \mathrm{vs} 117 \mathrm{mM}$ ), in order to match the composition of the perfusion fluid used in the study of skeletal muscle. As in previous studies, the fluid bathing the cardiac muscle in vitro contained $1 \mathrm{mM}$ $\mathrm{MgSO}_{4}$ and ventricles were equilibrated in the Ringer's for several hours before beginning measurements, to reduce spontaneous contraction. The $\mathrm{MgSO}_{4}$ was not used in the study of skeletal muscle but tests indicated that its presence and a longer equilibration period did not significantly alter the thermal dependence of contractility in the skeletal muscle. Thus, any differences in the contractile responses of the two tissues do not appear attributable to the slight differences in the methods by which they were tested.

In each muscle preparation, tension development typically remained maximal over a range of several degrees Celsius. To simplify comparative analysis of these data, the "optimal" temperature for muscle from each species was defined as the highest temperature at which tension development was maximal (i.e., the uppermost limits of the optimal temperature range).

\section{Evaluation of Thermal Adjustments of Intact Lizards}

Attention was focused on the thermal preferendum and heat resistance of each lizard studied in an attempt to relate temperature responses of the excised tissues 


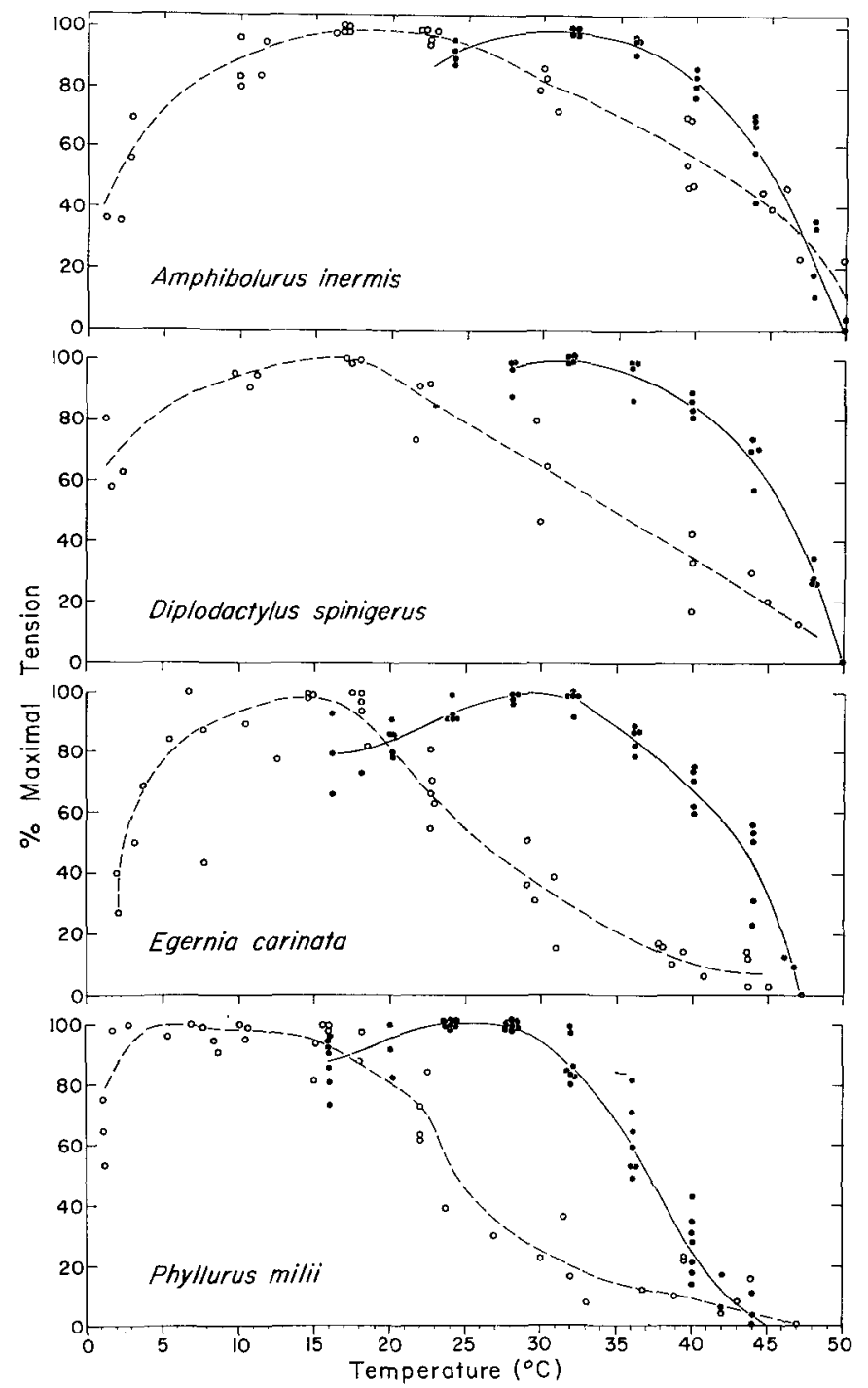

Fig. 1. Effects of temperature on isometric twitch tension development by excised skeletal muscle (shaded circles) and ventricular muscle (unshaded circle) from four species of lizards. Each point at a particular temperature or within a range of a few degrees represents the muscle from a separate individual; each tissue was tested over the entire range of temperatures shown for each species. Data for the two types of muscle are based on tissues taken from the same series of 4 to 7 individuals of each species although both muscles were not used in all cases (e.g., data are presented for 4 skeletal muscles but for only 3 ventricular preparations from Dipladactylus). Smooth curves were drawn through approximate mean values for each temperature. Muscles were stimulated to maximal twitch tension after a 5-minute equilibration period at each temperature and the data subsequently plotted on the basis of the maximal tension developed by the individual muscle over the entire range of temperatures tested 


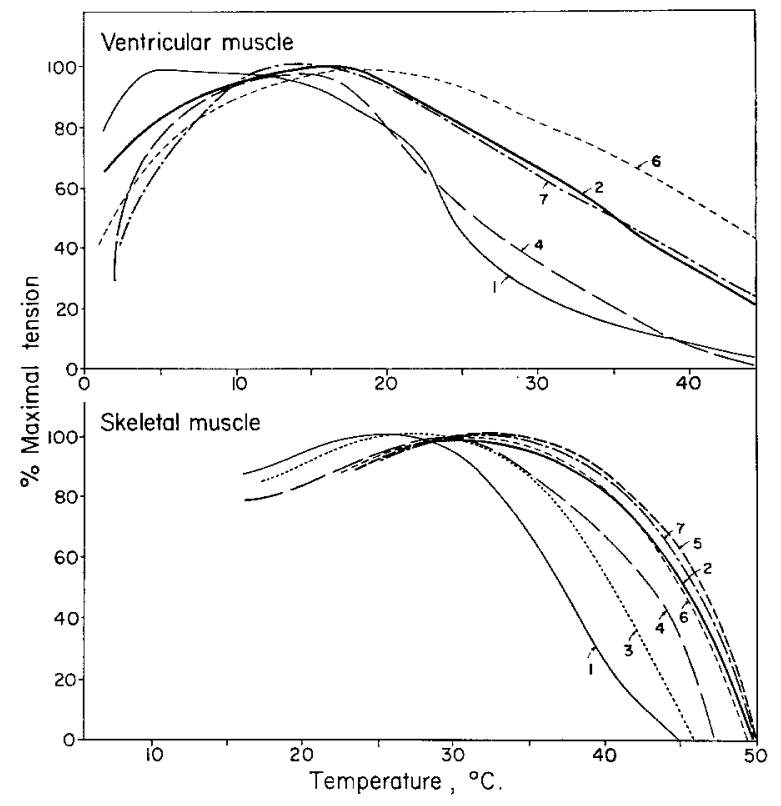

Fig. 2. Comparisons of the thermal responses in development of isometric twitch tension by excised ventricular and skeletal muscles from a variety of Australian lizards. The curves were based on data similar to those shown in Fig. 1, and show the approximate mean responses over the range of temperatures examined. The curves for each species are identified by numbers: 1 : Phyllurus milii; 2 : Diplodactylus spinigerus; 3: Gehyra punctata; 4: Egernia carinata; 5: Physignathus longirostris; 6: Amphibolurus inermis; 7 : Amphibolurus ornatus

and thermal adjustments at the organismal level. Thermal preferenda of intact individuals were taken to be those body temperatures maintained by lizards under conditions favorable for behavioral thermoregulation. These values were obtained from laboratory studies employing standardized thermal gradient chambers in which all of the Australian species used here have been tested (Licht et al., 1966a). It is recognized that the thermal preferendum of a species typically embraces a range of several degrees, but for convenience we have based comparisons on the mean body temperature maintained by species during test periods in the gradient chambers (mean preferred temperatures or MPT). Data regarding organismal heat resistance are based on studies of survival times of lizards at experimentally imposed high body temperatures (Licht et al., 1966 b).

\section{Results}

\section{Optimal Temperatures for Muscular Contractility}

The effects of temperature on the contractility of skeletal and cardiac muscles for four of the seven lizard species used are shown in Fig. 1. The variation shown in this graph is comparable to that observed for the other 


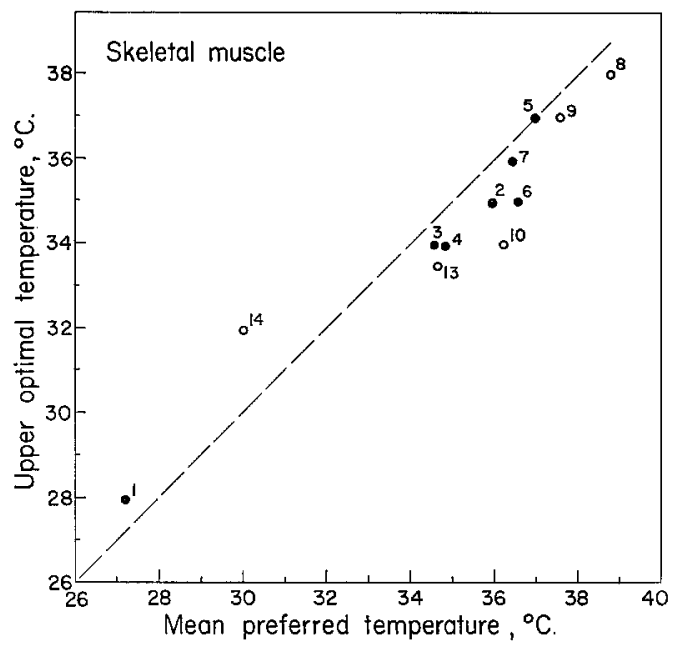

Fig. 3. The relation between the temperatures at which excised skeletal muscles develop maximal tensions (optimal temperatures) and the mean preferred body temperatures of the species from which they are taken. Values for optimal temperatures represent the highest thermal level at which twitch tension was still at its maximum. These values were obtained by interpolation from smooth curves drawn through available data (e.g., Figs. 1 and 2). Mean preferred temperatures are based on laboratory thermal gradient studies (LICHT et al., 1966a). Points represent individual species and are identified by numbers. Shaded circles correspond to the Australian species identified in Fig. 2; unshaded circles are North American species: 8: Dipsosaurus dorsalis; 9: Uma notata; 10: Sceloporus undulatus; 13: Eumeces obsoletus; 14: Gerrhonotus multicarinatus (data from LicHT, 1964)

species. The data indicate that marked differences exist in the optimal temperatures for contractility of the two types of muscle from the same individual; the cardiac muscle consistently developing maximal isometric twitch tension at a much lower temperature than the skeletal muscle. At temperatures where skeletal muscle tension was maximal (optimal temperatures), tension development in the cardiac muscle had declined to about $50 \%$ of its maximum. However, the slopes of the composite curves for the decline in tension at higher (supra-optimal) temperatures were much shallower in the cardiac than in skeletal muscle and complete loss of tension development (i.e., the lethal point) tended to occur at approximately the same temperatures in both muscles within the same individuals of a given species.

When the effects of temperature on muscle contractility are compared among all of the species examined (Fig. 2), considerable interspecific variation is evident in both types of muscle. Differences exist in the optimal ranges for contractility for each muscle, in the extent of decline in 


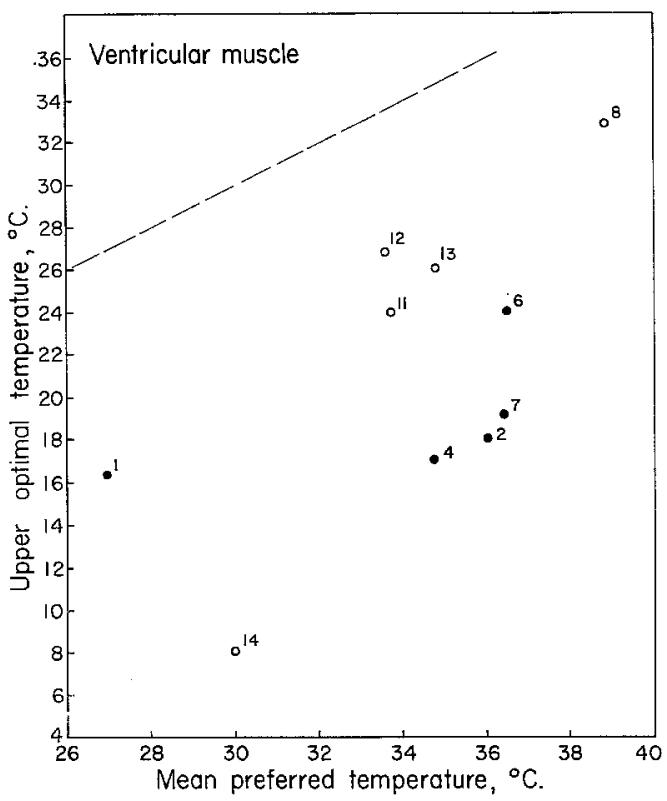

Fig. 4. The relation between the temperatures for maximal twitch tension development (optimal temperatures) for excised ventricular muscles and the mean preferred temperatures of the species from which they were taken. Data are presented as in Fig. 3. Additional North American species (unshaded circles) include: 11: Sceloporus occidentalis; 12 : Sceloporas jarrovi (data for all North American species are taken from DAWsoN, 1967)

tension at supra-optimal temperatures, and in upper lethal temperatures. Differences at suboptimal temperatures are less distinct, although some differences are evident among the ventricular tissues. A paucity of data at low temperatures in the case of skeletal muscles precludes detailed analysis for the performance of these tissues below their optima.

The optimal temperature for contractility in the skeletal muscle correlates closely with the mean preferred temperature in each species (Fig. 3). Furthermore, the absolute values for the optimal temperatures of this tissue are within a few degrees of the MPT in each species, as indicated in Fig. 3. Values obtained from previous studies of saurian skeletal muscle responses to temperature (see unshaded circles in Fig. 3) are in good agreement with the present data.

The optimal temperatures for development of tension by cardiac muscle also vary directly with MPT among the species of lizards that have been tested (Fig. 4). The order of these optimal temperatures roughly parallels that of the skeletal muscle for the species in which data for both tissues are available (cf. Figs. 3 and 4), the most notable exception being Eumeces 
obsoletus (No. 13 in the figures). However, the optimal temperature for cardiac muscle invariably falls about $15^{\circ} \mathrm{C}$ below the thermal preferendum of the species, in contrast to the fairly close agreement between the temperature for optimal contractility of skeletal muscle and the corresponding thermal preferendum of the species from which the tissue was obtained. Also, in Australian and American species having similar MPT's, samples of skeletal muscle tend to have similar optimal temperatures, whereas samples of cardiac muscle in some cases show wide discrepancies. That these discrepancies may be due to differences in methodology as outlined above rather than to inherent differences in cardiac tissues from the two groups cannot be discounted at this time.

\section{Thermostability of Muscle}

The heat resistance of the skeletal muscles was judged by the extent of irreversible damage occurring at various temperatures within 5 minutes and by the lethal temperatures (i.e., the temperature at which the muscle first failed to respond to stimulation, and contractility was irreversibly lost after the 5-minute exposure). During analysis of the muscle preparations, the muscles were tested at the reference temperature $\left(24^{\circ} \mathrm{C}\right)$ after exposure to each high temperature, before proceeding to the next higher temperature. A marked drop in the tension developed at this reference temperature was taken to indicate that irreversible damage had occurred during the 5-minute exposure to the preceeding high temperature. Since increments of $2-4^{\circ} \mathrm{C}$ were generally used in this study (see Fig. 1), the exact point of irreversible damage can only be approximated. Reference tensions often fluctuated by several per cent even after exposure at low temperatures; thus, for comparative purposes, a drop in reference tension of more than $20 \%$ of the initial was taken as an indication of significant damage. The approximate high temperatures found to cause such damage to skeletal muscle in each individual and their relations to other aspects of tissue and organismal function are shown in Fig 5 .

In each species, significant irreversible damage (as defined above) occurred approximately $10-14^{\circ} \mathrm{C}$ above the uppermost limits of the temperature range for maximal contractility. Comparison of Figs. 1 and 5 show that the development of tension decreased by about $75 \%$ before reaching the temperature that caused such irreversible damage. Thus, it appears that most of the decline in the tension developed by skeletal muscles above the optimal temperature represents a reversible suppression of contractility. In general, the lethal temperature of the skeletal muscle is about $2^{\circ} \mathrm{C}$ above the temperature where this irreversible damage is first detected, indicating a high temperature coefficient for the irreversible loss of contractility. 


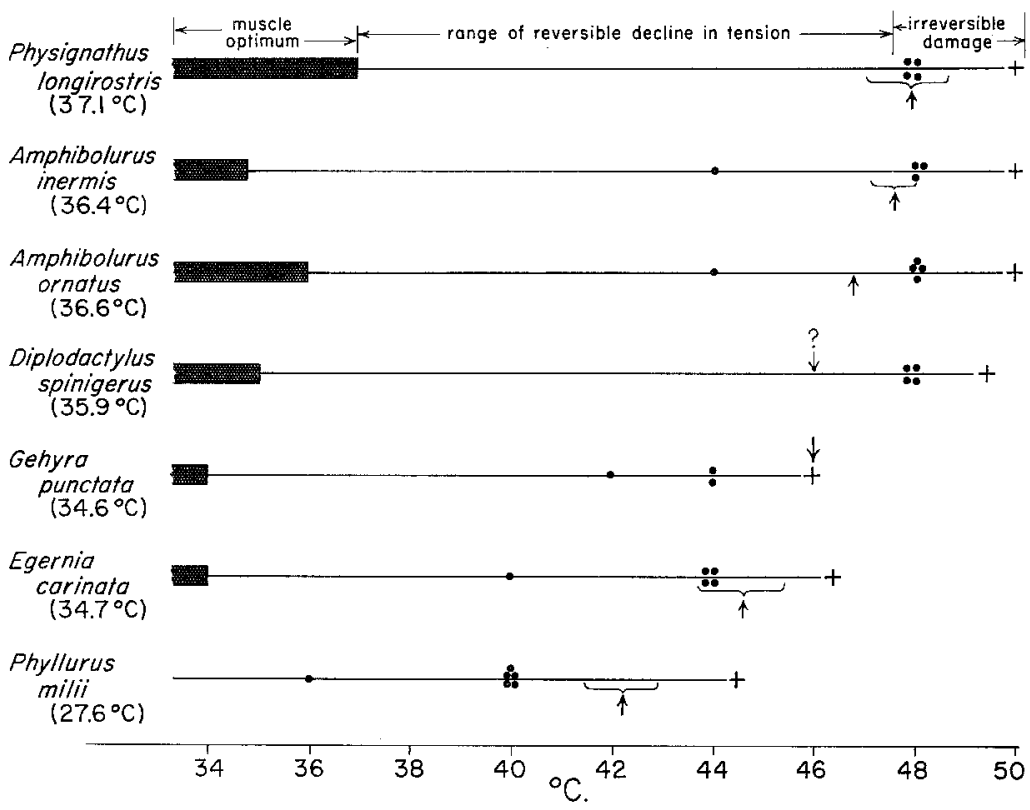

Fig. 5. Summary of the relationship between various aspects of tissue (skeletal muscle) and organismal temperature responses in 7 species of Australian lizards. The mean preferrred body temperature for each species is shown in parentheses at the left. To the right of each species are shown, in order, the range of temperatures where tension development was maximal for the species skeletal muscles (heavy bar), where muscle tension development declined but where recovery occurred when transferred to a lower temperature, and where the decline in tension was irreversible (see example at top). The solid points near the right end of each scale represent the temperatures where irreversible damage was first noted in individual muscles, the arrow and parentheses indicate the uppermost temperatures at which the species can survive for about 5 minutes (based on LICHT et al., $1966 \mathrm{~b}$ ), and the crosses at the far right mark the lethal temperatures for the muscles (where no contraction occurred afterafive minute equilibration period)

In a previous report (LICHr, 1964), it appeared that the temperature at which irreversible damage occurs in saurian skeletal muscle coincides closely with the upper lethal temperature of the species. The present study indicates a greater variability in the relation between organismal and tissue heat resistance. Inaccuracies in the methods for determining the two aspects of heat resistance preclude detailed comparison, especially in view of the high temperature coefficients for such processes. However, a generally good correlation exists between the extent of organismal heat resistance and that of the muscles.

In ventricular muscles irreversible damage generally did not appear until shortly before the upper lethal temperature was reached; this 
corresponded closely to the upper lethal temperature for the skeletal muscle from the same species (see Fig. 5). Thus, the gradual decline in tension development in the cardiac muscle at supra-optimal temperatures was probably also largely due to reversible processes.

\section{Diseussion}

The data presented in this report provide additional evidence for the existence of interspecific divergence in several aspects of the thermal relations of saurian cardiac and skeletal muscles, including their performance in normal ranges of temperature (i.e., optimal temperatures) and in their resistance to inactivation by high temperatures (i.e., thermostability). Furthermore, comparison of cardiac and skeletal muscle from single individuals confirms that these two types of muscle exhibit distinct responses in vitro with regard to their temperature optima for isometric twitch tension development. The performance of the skeletal muscle under our assay conditions appears more closely related to the normal "activity" temperatures (see below) of the organism, but it is difficult to evaluate differences in responses of excised tissue in terms of in vivo organismal performance. These differences point up the need for examining a variety of tissues before attempting to relate absolute values from in vitro studies to the performance of tissues in vivo. The feature of these data that we wish to emphasize is the similarity of the interspecific divergence evident in both skeletal and cardiac muscles. The parallel divergence suggests that both tissues are similarly involved in the physiological adjustment of lizards to temperature.

The question of the stability of tissue thermal responses must be considered before interspecific variations in these responses can be evaluated. Unfortunately, little evidence is presently available concerning the extent to which the function of individual tissues may vary temporally as a result of phenotypic modification by acclimation or other ontogenic influences. UsHakov (1964) reported a slight seasonal shift in the thermostablity of skeletal muscles from one species of lizard, Phrynocephalus helioscopus; this shift was the reverse of that expected on the basis of seasonal climatic changes and was thought to be related to hormonal changes associated with the reproductive cycle. In preliminary studies concerning thermal acclimation in the lizard Uma notata, skeletal muscles taken from animals acclimated to 16,25 or $38^{\circ} \mathrm{C}$ for 3 weeks exhibited the same optimal temperatures and thermostabilities (LICHT, 1967). These observations suggest that the major interspecific variations in muscular thermal responses noted in this and related reports cannot be explained by ontogenetic circumstances such as differences in acclimation, reproductive status, etc. 
Interspecific divergence in the thermal adjustments of the two kinds of muscle cannot be accounted for solely by variations in geographical (i.e., climatic) distribution, general activity patterns (e.g., nocturnality vs. diurnality), or phylogenetic relations. For example, despite marked differences in their muscles, all of the geckos, several of the agamids, and the skink studied here are sympatric and can be collected within the same general habitats in Western Australia. In contrast, several species in which muscles show similar thermal responses (e.g., Australian agamids and New World iguanids) occur in widely diverse habitats and climates. All three geckos examined are crepuscular or nocturnal, yet their tissues are very divergent with regard to thermal responses: the muscle of one species represent the least heat resistant with the lowest optimal temperature while those of the other two are among the most resistant, matching those of diurnal species of skinks, agamids, and iguanids.

\section{Optimal Temperatures}

It is now widely recognized that lizards are able to exercise considerable control over their body temperature by means of behavioral thermoregulation and various physiological adjustments. The strong correlation between the thermal optima for muscles and the lizards' thermal preferenda (the characteristic range of body temperature maintained when provided with a wide choice of thermal conditions) indicates that thermoregulatory behavior represents a fundamental feature of thermal adaptation among lizards. For example, the similarity of tissue adjustments to temperature among agamids and iguanids is consistent with the observation that these animals tend to regulate at similar body temperatures, despite wide differences in their distribution and in the macroclimatic conditions that they encounter (Lichт et al., 1966a).

The thermal divergence among geckos represents a particularly interesting example of the importance of thermoregulatory behavior. Even though all are nocturnal, studies in laboratory thermal gradient chambers indicated a marked divergence in the body temperatures preferred by the three species (Lіснт et al., 1966a). Some preferred temperatures as high as most diurnal species, whereas others showed much lower preferences. Field observations have shown that those nocturnal species with unusually high preferred temperatures are indeed able to elevate body temperatures to these levels by moving selectively within their diurnal retreats, although they are typically active at much lower body temperatures $\left(5-20^{\circ} \mathrm{C}\right.$ below preferenda) when abroad at night. Comparative studies of the responses of their muscles to temperature in vitro suggest that the performance of these tissues is more closely related to thermal preferenda than to the normal nocturnal activity temperatures. 


\section{Thermostability}

The divergence in heat resistance of skeletal muscles among lizards generally parallels that seen in the optimal temperatures for tension development. This relationship is consistent with the observation that organismal heat resistance tends to parallel thermal preferenda among lizards; i.e., the more thermophilic species, as judged by their thermal preferenda, tend to have higher lethal temperatures (Licht et al., 1966 b). However, organismal heat resistance is more difficult to assess than thermal preferenda, because of the importance of the time factor in thermal injury. Furthermore, the heat resistance of an individual lizard appears to be more readiliy modified than thermal preferenda (LiCHT, 1968).

UsHakov and his co-workers (see reviews by UsHaKov, 1964, 1966) have examined the heat resistance of a wide variety of tissues and enzymes, including observations on saurian skeletal muscles. Finding that heat resistance of individual tissues or enzymes often far exceeds that of the whole organism, UsHaKov (1966) suggested that changes in thermostability of enzymes or tissues might be of secondary importance to their correlation with the performance of the tissues at normal temperatures. Thus, knowledge of optimal temperatures would be more important than that of the thresholds for injury. The parallel between optimal temperatures for tissue performance and tissue lethal temperatures observed in cardiac and skeletal muscles supports the view that variations in heat resistance generally reflect differences in tissue capacities at more "normal" temperatures. However, thermal adjustments of diverse tissues cannot be readily compared on the basis of a single parameter, since, despite similarities in heat resistance the optimal temperatures for one aspect of cardiac and skeletal muscle contractility are very distinct.

It appears, in the case of both types of saurian muscles examined, that absolute values for heat resistance of the tissue, (recognizing the limitations of predicting in vivo performance from in vitro studies) may be closely related to general organismal heat resistance. Particularly important is the suggestion that even brief periods of activity at temperatures exceeding the thermal preferendum could lead to irreversible damage to the muscles.

Our values favorably agree with the extensive data on muscle thermostability reported by Usinaxov $(1960,1964,1966)$ wherever data is available for thermal preferenda on the species studied. This agreement suggests that thermal divergence in skeletal muscle in a manner correlated with thermal preferenda exists in a wide range of saurian species representing many different families.

UsHakov has stressed the geographical distribution of species to explain observed divergence in tissue and enzyme heat resistance, except where special behavioral patterns, such as nocturnalism, are involved. It 
may be inaccurate to include all poikilotherms in a common conceptual scheme with regard to environmental thermal relations, since not all exercise the same high degree of behavioral control over body temperatures evident in lizards. Since species (or subspecies) living in diverse habitats often maintain very similar body temperatures (i.e., utilize relatively similar microhabitats), while sympatric species in the same macro-environment may operate at distinct body temperatures during activity, geographical distribution could give very misleading impressions of the thermal conditions for which lizards have become adapted. The thermal conditions imposed by the species thermal preferenda should be taken into account.

The divergence in thermal adjustments of muscles is apparently only one aspect of the cellular and subcellular changes that underlie thermal adjustments in lizards. There is some evidence for similar interspecific variations in thermal responses in nerves and it seems likely that other somatic tissue functions would show similar variations (UsHaKov, 1964, 1966). Interspecific divergence similar to that found in muscle tissue is also evident in reproductive tissues (germinal epithelium) of lizards (LICHT and BAsU, 1967). The basis for these variations at the tissue level is not well understood, but it is probably associated with differences in the thermal adjustments of individual enzymes. For example, interspecific variations in skeletal muscles observed here correlate well with variations in optimal temperatures and thermostabilities previously demonstrated in the muscle myosin ATPase of these species (Lіснт, 1967). In all cases examined, cthe divergence at the tissue and subcellular levels correlate well with the thermal preferenda of the species examined.

Acknowledgements. We are grateful to Professor H. WARING and Professor A. R. MaIN of the University of Western Australia for providing facilities for this work and for their numerous courtesies during our visit in their department. Our work was supported in part by National Science Foundation Grant G-23137, the Guggenheim Foundation, and Horace H. Rackham School of Graduate Studies to W. R. Dawson.

\section{References}

Dawson, W. R.: Physiological responses to temperature in the lizard Eumeces obsoletus. Physiol. Zool. 33, 87 (1960).

- Interspecific variation in physiological responses of lizards to temperature. In: W. W. Milstead (ed.), Lizard Ecology Symposium, Univ. Mo. Press, Columbia, p. 230 (1967).

- and G. A. Bartholonew: Metabolic and cardiac responses to temperature in the lizard Dipsosaurus dorsalis. Physiol. Zool. 31, 100 (1958).

Lichт, P.: A comparative study of the thermal dependence of contractility in saurian skeletal muscle. Comp. Biochem. Physiol. 13, 27 (1964).

- Thermal adaptation in the enzymes of lizards in relation to preferred body temperatures. In: Molecular Mechanisms of Temperature Adaptation. p. 131. London-New York: Academic Press 1967. 
Lтснт, P.: Response of the thermal preferendum and heat resistance to thermal acclimation under different photoperiods in the lizard Anolis carolinensis. Amer. Midland Nat. 79, 149 (1968).

- and S. L. BASU: Influence of temperature on lizard testes. Nature (Lond.) 213, 672 (1967).

-, W. R. Dawson, V. H. Shommaker, and A. R. Main: Observations on the thermal relations of Western Australian lizards. Copeia 1966, 97 (1966a).

- - Heat resistance of some Australian lizards. Copeia 1966, 162 (1966 b).

Usнakov, B. P.: Eine zytophysiologische Analyse der Anpassung der Reptilien an das Leben in der Wüste Karakum: Zool. Jb. 87, 507 (1960).

- Thermostability of cell and proteins of poikilotherms and its significance in speciation. Physiol. Rev. 44, 518 (1964).

- The problem of associated changes in protein thermostability during the process of speciation. Helgoländer wiss. Meeresunters. 14, 466 (1966).

Dr. PaUl LichT

Department of Zoology

University of California

Berkeley, Calif. 